\title{
Blickdiagnose Plazenta
}

\author{
Kerstin Steiner und Rahel Thiessat, Hebammen, Stuttgart
}

\section{Bei diesen Blickdiagnosen muss die Plazenta ganz besonders kritisch auf Vollständigkeit geprüft werden.}

„....nun muß zunächst die Nachgeburt genau besichtigt werden nach Entfernung oberflächlicher anhaftender Blutgerinnsel mit der Hand. Man lege sie auf eine flache Unterlage und achte vor allem darauf, ob die schiefergrau spiegelnde Gebärmutterfläche des Mutterkuchens unverletzt ist, ob nicht etwa ein Stück fehlt. Auch den Rand prüfe die Hebamme daraufhin; sie sehe ferner durch Halten der Eihäute gegen das Licht am Rand des Kuchens nach, ob nicht etwa ein Blutgefäß über ihn hinaus in der Lederhaut verläuft, das würde Verdacht auf einen zurückgebliebenen $\mathrm{Ne}$ benkuchen erwecken. Auch die Eihäute prüfe sie auf Vollständigkeit.“

So steht es im „Lehrbuch der Hebammenkunst" von Dr. B. S. Schultze, 16. Auflage von 1928.

Trotz unseres medizinischen Fortschritts ist es auch heutzutage noch eine Handwerkskunst, die Plazenta nach ihrer Geburt bzw. manuellen Entwicklung zu beurteilen. Es ist wichtig, sich ausreichend Zeit zu nehmen für die Prüfung auf Vollständigkeit.

Voraussetzung für die Inspektion sind ausreichend Platz, eine glatte ebene Unterlage, ausreichende Lichtverhältnisse.

\section{Leitfragen bei der Inspektion}

\section{Kindliche Plazentaseite}

- Sind die Eihäute vollständig?

- Gibt es abirrende Gefäßverläufe, die offen in den Eihäuten enden?

\section{Mütterliche Plazentaseite}

- Ist die perlmuttartig glänzende Oberfläche der Dezidua unverletzt?

- Gibt es sichtbar fehlende Plazentastücke?

- Gibt es blutende Stellen?

- Bedecken die Eihäute mindestens 2/3 der Plazentaseite? (So kann davon ausgegangen werden, dass diese vollständig sind.)

- Randbeurteilung: Ist der Plazentarand durchgehend in Verbindung der Zotten mit den Eihäuten, gibt es auffallende Lücken?

\section{Besonderheiten}

- In den Eihäuten verlaufende Gefäße: ist ein Anfang bzw. ein Ende des Gefäßverlaufes sichtbar?

- Bei Nebenplazenten: sind diese klar begrenzt sichtbar mit Verbindung zur Hauptplazenta?

- Nach manueller Plazentalösung: oftmals sind hier Strukturen durch die Manipulation auseinander gerissen. Fügen sich alle Plazentateile passend zusammen und bilden ein einheitliches Bild?
- Mehrlinge: Gibt es eine oder mehrere Plazenten? Wie viele Eihäute gibt es?

\section{Weitere Fragen}

- Wie schwer ist die Plazenta?

- Wie sind die Farbe und der Geruch der Plazenta?

- Passt die Größe der Plazenta zur Größe des Kindes und zur SSW?

- Gibt es Koagelbildung? (als Hinweis auf vorzeitige Plazentalösung im Geburtsverlauf )

- Gibt es Verkalkungen, sichtbare Zysten, Verhärtungen, Infarkte, Fettinfarkte?

\section{Nabelschnur}

- Nabelschnuransatz: zentral, lateral, marginal auf der kindlichen Plazentaseite

- Nabelschnuransatz in den Eihäuten?

- Dicke und Länge der Nabelschnur?

- Gibt es Formveränderungen?

- Gibt es Nabelschnurknoten?

- Sind drei Gefäße in der Nabelschnur (eine Vene, zwei Arterien) sichtbar?

Neben der medizinischen Beurteilung der Plazenta und der Nabelschnur ist noch etwas anderes wichtig: sich Zeit nehmen, um den Eltern dieses Wunderwerk zu zeigen, zu erklären und sie es ebenfalls berühren zu lassen. 
Für uns ist die Plazenta mit Nabelschnur etwas Alltägliches. Nicht so für die Eltern. Es gibt viele Beschreibungen zur Bedeutung der Plazenta und der begleitenden Rituale in den verschiedenen Kulturen. Dem Fetus stand die Plazenta über Wochen zur Verfügung, als einziges Überlebensmittel, es ist die vertraute Verbindung zur Mutter, mit der Nabelschnur konnte es spielen.

In der Vorstellung vieler Eltern ist die Plazenta blutig und ekelerregend, weshalb sie sie oft gar nicht sehen wollen. Wenn wir uns die Zeit nehmen, die Plazenta zu säubern und den Eltern ihre Schönheit als Lebensbaum näher zu bringen, dann bekommt dieses Wunderwerk seine Bedeutung und Achtung, die es verdient

\section{Placenta annularis (Ringplazenta)}

Blickdiagnose: Gürtel- oder ringartige Form der Plazenta (Abb. 1-4)
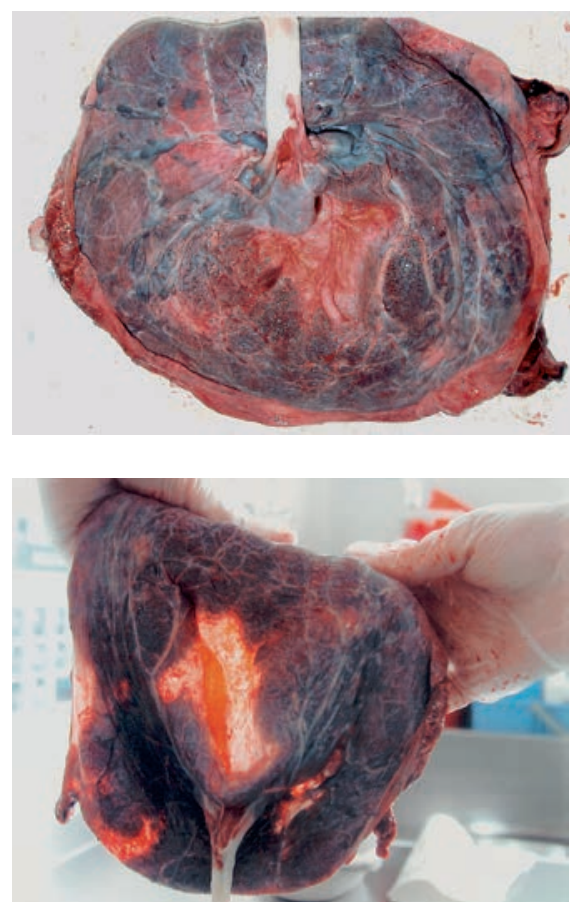

Abb. 1 und 2 „Fehlender“ Plazentaanteil von der kindlichen Seite der Plazenta aus betrachtet.

\section{Pathophysiologie}

Formanomalie der Plazenta mit Untergang örtlicher Chorionzotten oder unvollständiger „Chorionglatzenbildung“. Sie kann durch Endometriuminsuffizienz am Nidationort entstehen. Sehr selten.

Klinisches Bild: In der Mitte der Plazenta ist eine „freie“ Stelle sichtbar.

\section{Placenta succenturiata}

Blickdiagnose: Nebenplazenta (Abb. 5-7)

\section{Pathophysiologie}

Kleiner Zottenkomplex, der sich in einiger Entfernung zur Hauptplazenta auf dem Chorion leve entwickelt. Er ist durch Gefäße, welche über die Eihaut verlaufen, mit der Hauptplazenta verbunden.

Häufigkeit: bei bis zu 3\% aller Geburten.

Klinisches Bild: Eine oder mehrere Nebenplazenten
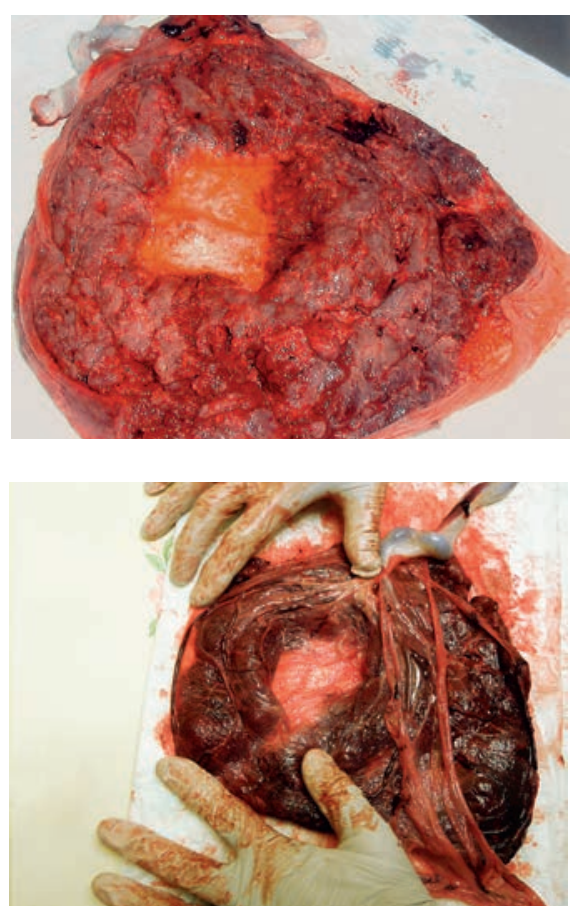

Abb. 3 und 4 „Fehlender“ Plazentaanteil von der mütterlichen Seite der Plazenta aus betrachtet.
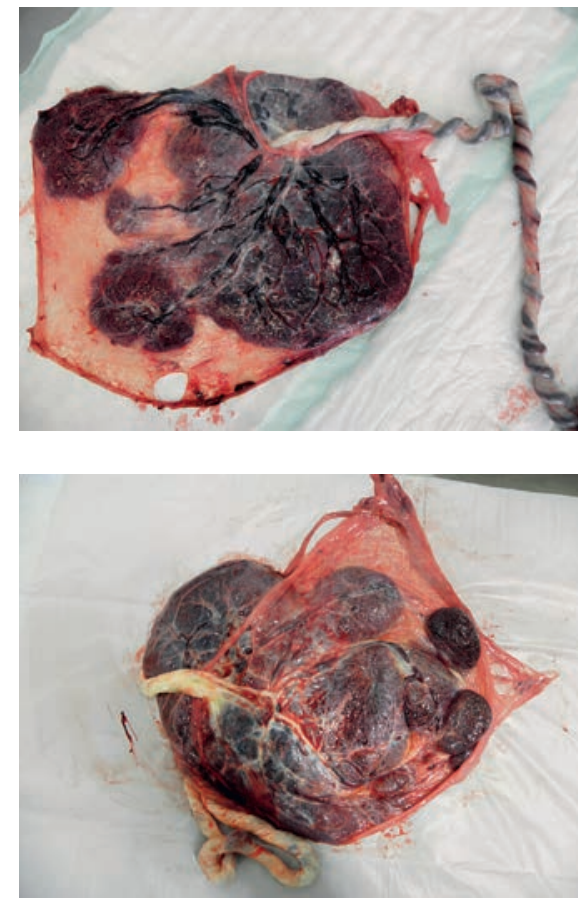

Abb. 5 und 6 Auf der kindlichen Plazentaseite sind kleine Zottenkomplexe mit Gefäßverbindungen zur Hauptplazenta erkennbar.

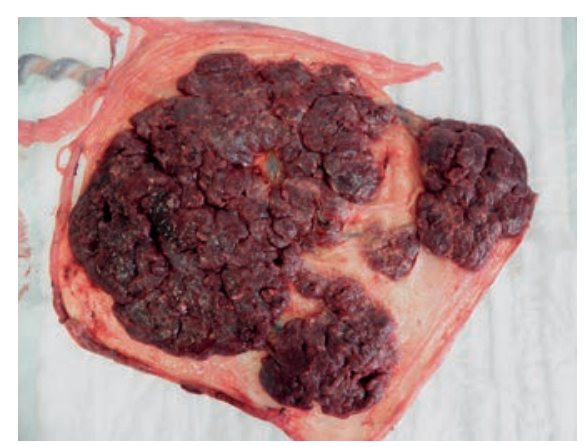

Abb. 7 Ansicht von der mütterlichen Plazentaseite aus. 


\section{Placenta bipartita}

Blickdiagnose: Zwei ähnlich große Plazenten (Abb. 8-14)

\section{Pathophysiologie}

Im Normalfall entwickelt sich die Plazenta als fast runde Fläche um die Stelle der Implantation der Blastozyste (befruchtete Eizelle). Der Nabelschnuransatz an der Plazenta (Insertion) ist zentral (mittig). Lokale, endometrium- oder chorionbedingte Störungen der Einnistung der befruchteten Eizelle (Nidation) und des Wachstums der Plazenta können zu unterschiedlichen Plazentaformen und Insertionen der Nabelschnur führen.

Klinisches Bild: Zwei ähnlich große Plazenten befinden sich auf der Eihaut. Die Insertion der Nabelschnur liegt in der zottenfreien Zone zwischen den Plazenten oder auf einer der beiden Plazenten, wobei Nabelschnurgefäße zur anderen Plazenta über die Eihaut verlaufen. Fällt meist erst nach der Geburt bei der Inspektion der Plazenta auf.

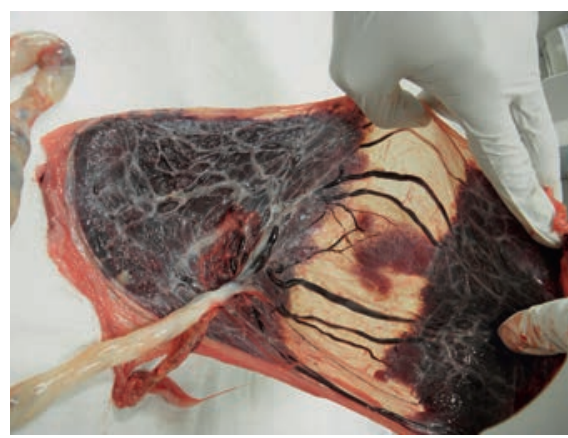

Abb. 8 Zwei fast gleich große Plazentaanteile bei einer Einlingsschwangerschaft mit parallel verlaufenden Gefäßverbindungen durch die Eihäute (Ansicht der kindlichen Plazentaseite).

Alle Abb. aus Kerstin Steiner (2013): Blickdiagnosen in Geburtshilfe und Neonatologie. Hippokrates Stuttgart.

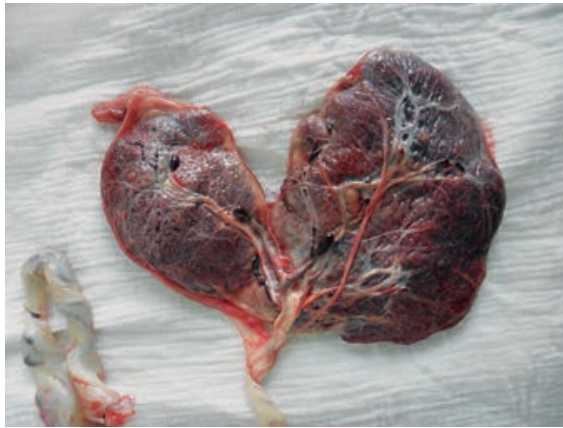

Abb. 9 Zwei unterschiedlich große Plazentaanteile mit marginalem Nabelschnuransatz (kindliche Plazentaseite). Im kleineren Anteil sind leichte Verkalkungen zu erkennen.

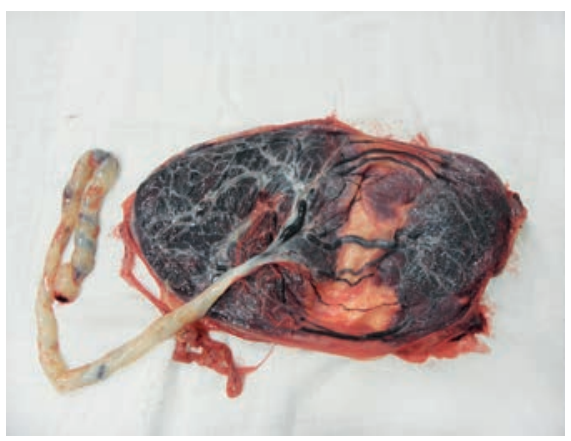

Abb. 10 Zwei unterschiedlich große Plazentaanteile mit frei verlaufenden Gefäßen in den Eihäuten (Ansicht der kindlichen Plazentaseite).

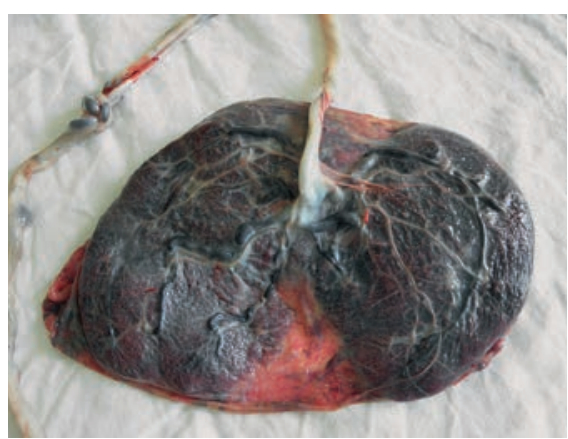

Abb. 11 Zentraler Nabelschnuransatz zwischen zwei fast gleich großen Plazentaanteilen ohne frei verlaufende Gefäße.

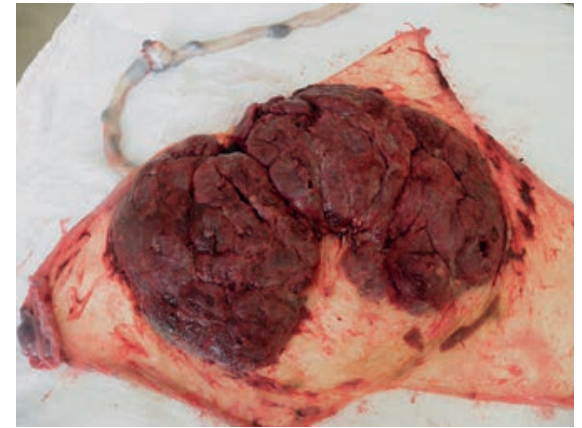

Abb. 12 Ansicht der mütterlichen Plazentaseite (gleiche Plazenta wie in Abb. 11).

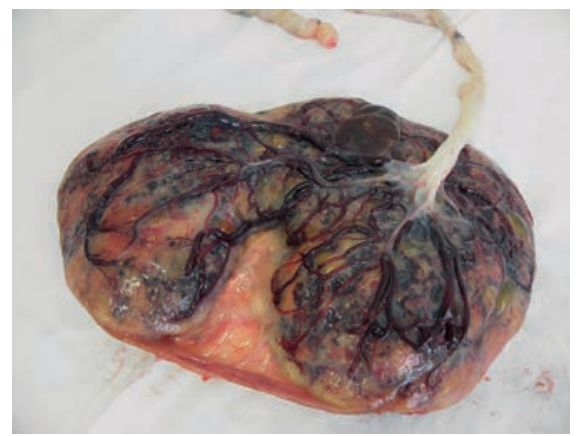

Abb. 13 Das Bild zeigt zwei Plazentaanteile, der Nabelschnuransatz befindet sich auf einem Teil. Es gibt keine frei verlaufenden Nabelschnurgefäße. In der Plazenta sind reichlich Fettinfarkte zu erkennen. Es handelt sich um eine rasche Spontangeburt in der 37. SSW.

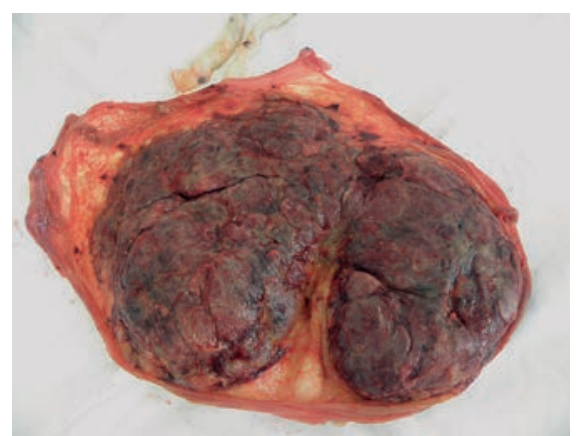

Abb. 14 Ansicht der mütterlichen Plazentaseite (dieselbe Plazenta wie in Abb. 13).

\section{Kerstin Steiner}

Hebamme

E-Mail: kstoni@gmx.de 